\title{
Association of Complement Receptor 2 Gene Polymorphisms with Susceptibility to Osteonecrosis of the Femoral Head in Systemic Lupus Erythematosus
}

\author{
Tae-Ho Kim, ${ }^{1,2}$ Sang-Cheol Bae, ${ }^{3}$ Sang-Han Lee, ${ }^{4}$ Shin-Yoon Kim, ${ }^{2,5}$ and Seung-Hoon Baek ${ }^{5}$ \\ ${ }^{1}$ Biomedical Research Institute, Kyungpook National University Hospital, 135 Dongdeok-ro, Jung-gu, Daegu 700-721, Republic of Korea \\ ${ }^{2}$ Skeletal Diseases Genome Research Center, Kyungpook National University, 135 Dongdeok-ro, \\ Jung-gu, Daegu 700-721, Republic of Korea \\ ${ }^{3}$ Department of Rheumatology, Hanyang University Hospital for Rheumatic Diseases, 17 Haengdang-Dong, \\ Seongdong-Gu, Seoul 133-792, Republic of Korea \\ ${ }^{4}$ Department of Food Science \& Biotechnology, Kyungpook National University, 80 Daehak-ro, Buk-gu, \\ Daegu 41566, Republic of Korea \\ ${ }^{5}$ Department of Orthopedic Surgery, Graduate School of Medicine, Kyungpook National University, \\ 130 Dongdeok-ro, Jung-gu, Daegu 700-721, Republic of Korea
}

Correspondence should be addressed to Seung-Hoon Baek; sbaek@knu.ac.kr

Received 10 March 2016; Accepted 6 June 2016

Academic Editor: Hai-Feng Pan

Copyright (C) 2016 Tae-Ho Kim et al. This is an open access article distributed under the Creative Commons Attribution License, which permits unrestricted use, distribution, and reproduction in any medium, provided the original work is properly cited.

Osteonecrosis of the femoral head (ONFH) is a complex and multifactorial disease that is influenced by a number of genetic factors in addition to environmental factors. Some autoimmune disorders, including systemic lupus erythematosus (SLE), rheumatoid arthritis (RA), and inflammatory bowel disease (IBD), are associated with the development of ONFH. Complement receptor type 2 (CR2) is membrane glycoprotein which binds C3 degradation products generated during complement activation. CR2 has many important functions in normal immunity and is assumed to play a role in the development of autoimmune disease. We investigated whether CR2 gene polymorphisms are associated with risk of ONFH in SLE patients. Eight polymorphisms in the CR2 gene were genotyped using TaqMan ${ }^{\mathrm{TM}}$ assays in 150 SLE patients and 50 ONFH in SLE patients (SLE_ONFH). The association analysis of genotyped SNPs and haplotypes was performed with ONFH. It was found that three SNPs, rs3813946 in $5^{\prime}$-UTR (untranslated region), rs311306 in intron 1, and rs17615 in exon 10 (nonsynonymous SNP; G/A, Ser639Asn) of the CR2 gene, were associated with an increased risk of ONFH under recessive model ( $P$ values; 0.004 0.016). Haplotypes were also associated with an increased risk (OR; 3.73 ) of ONFH in SLE patients. These findings may provide evidences that CR2 contributes to human ONFH susceptibility in Korean SLE patients.

\section{Introduction}

Osteonecrosis of the femoral head (ONFH) is a complex and multifactorial disease which can be affected by combined genetic factors with relatively small effect in addition to environmental factors [1]. A variety of conditions, such as use of corticosteroids, alcohol abuse, and rheumatic diseases were reported as risk factors for secondary ONFH. Among autoimmune diseases, systemic lupus erythematosus (SLE) has shown higher incidence of ONFH ranging from 5 to $30 \%$, than that of general population and ONFH, in turn, results in significant morbidity [2]. Although corticosteroid use has been reported as a significant predictive factor for developing ONFH in patients with SLE [3], there are also reports of patients with SLE complicated by ONFH, who have not taken corticosteroid $[4,5]$. This implicates possible role of the disease progression itself or underlying genetics. Although some studies reported that immunologic factors including 
interleukins and tumor necrosis factors might develop ONFH $[6,7]$, most genetic studies have focused on gene polymorphisms affecting the coagulation and fibrinolytic systems $[8$, 9]. Moreover, few genetic studies were performed to reveal their roles in the development of SLE_ONFH [10].

Human complement receptor 2 (CR2) is encoded by a single gene containing 20 exons, which is located at chromosome 1q32.2 [11] and is expressed on mature B or follicular dendritic cells (FDCs) [12]. CR2 was known to bind C3 degradation products generated during the process of complement activation [13] and some studies suggested that CR2 might play an important role in immunity [14]. Therefore, given the pleiotropic effects of complement receptor, we investigated whether polymorphisms of the CR2 gene are associated with the susceptibility of SLE_ONFH.

\section{Material and Methods}

2.1. Patients and DNA. Blood samples and medical records were obtained from 150 SLE patients (13 males, 137 females; mean age, $31.37 \pm 9.97$ ) and 50 SLE_ONFH patients (4 males, 46 females; mean age, $31.28 \pm 9.04)$. The SLE and SLE_ONFH patients were consecutively recruited from the Hanyang University Hospital for Rheumatic Diseases (Seoul, Korea). All SLE patients met the American College of Rheumatology (ACR) criteria for the classification of SLE [15]. Genomic DNA was isolated from the peripheral blood of each participant using a FlexiGene DNA Kit (QIAGEN, Valencia, CA). The current study was approved by the Institutional Review Board, and all participants in this study provided their informed consent.

2.2. Genotyping. Eight single nucleotide polymorphism (SNP) sites in the CR2 gene were selected based on locations, potential relevance to disease, and published data $[11,16$, 17]. The genotype was determined using a TaqMan fluorogenic $5^{\prime}$-nuclease assay with predesigned or custom TaqMan primer/probe sets (Applied Biosystems, Foster City, CA). For genotyping of polymorphic sites, amplification primers and probes were designed for TaqMan assays (Applied Biosystems, Foster City, CA). The primer and probe sequences are indicated in Table 1. We designed both the PCR primers and the minor groove binder (MGB) TaqMan probes using Primer Express (Applied Biosystems). All reactions were performed following the manufacturer's protocol. Details regarding the PCR reaction and TaqMan assay have been described previously [9]. The fluorescence data files from each plate were collected and analyzed using automated allele-calling software (SDS 2.2, Applied Biosystems).

2.3. Statistical Analyses. The threshold of Hardy-Weinberg equilibrium (HWE) $P$ value is set at $>0.05$. Allelic or genotype association tests in the case-control were calculated using $\chi^{2}$ test or Fisher's exact test. Odds ratios (ORs) and corresponding 95\% confidence intervals (CIs) for case-control data were also calculated. Genotypes were given codes of 0,1 , and $2 ; 0,1$, and 1 ; and 0,0 , and 1 in the codominant, dominant, and recessive models, respectively. The strength of linkage disequilibrium (LD) among the pairs of SNPs was evaluated using Haploview 4.2 software (http://www.broad.mit.edu/mpg/haploview/). Haploview software was also used to calculate haplotype structures and their frequencies within LD blocks. Haplotypes with frequencies $<5 \%$ were excluded from the following analysis. Continuous variables were compared by Student's $t$ test or ANOVA. All analyses were two-tailed, and $P$ values $<0.05$ were considered to be statistically significant.

\section{Results}

3.1. Genetic Association of CR2 SNPs with SLE_ONFH Susceptibility. To determine whether CR2 gene polymorphisms might contribute to the susceptibility of ONFH development in SLE patients of Korea (SLE_ONFH), the sample of 150 SLE and 50 SLE_ONFH Korean patients was genotyped using eight SNPs spanning a $39 \mathrm{~kb}$ region of the CR2 gene from $0.6 \mathrm{~kb}$ upstream to $2.8 \mathrm{~kb}$ downstream of the gene (Figure 1). We selected 8 informative SNPs that included 1 regulatory SNP (rs3813946 in $5^{\prime}$-UTR; T/C), 1 exonic SNP (rs17615 in exon 10; G/A, Ser639Asn), and 6 haplotypetagging intronic SNPs that tagged the two haplotype blocks (Figure 1(b)). The resulting SNP data including location, amino acid substitution, genotype, MAF, and HWE of all analyzed polymorphisms are demonstrated in Table 2. Table 3 shows a comparison of genotype frequencies between casecontrol groups. When genotype distributions between the SLE (control) and SLE_ONFH (case) groups were compared, the SNPs rs3813946 in 5' -UTR (untranslated region), rs311306 in intron 1, and rs17615 in exon 10 (nonsynonymous SNP; G/A, Ser639Asn) of the CR2 gene, located in block 1, demonstrated the evidence for association with risk of ONFH under recessive model ( $P$ values; $0.004 \sim 0.016)$. None of block 2 SNPs showed evidence for association (Table 3 ).

\subsection{Association of CR2 SNP Haplotypes with SLE_ONFH} Susceptibility. Because LD is believed to be highly structured, with conserved blocks of sequence separated by hotspots of recombination, the function of a conserved haplotype may result from interaction between polymorphisms within a block. Therefore, SNP haplotypes were then constructed on the basis of genotypes of the SNPs, which resided in LD block (Figure 1(b)). Four major haplotypes with frequencies $>0.05$ were predicted in LD block 1 , and the frequency of each haplotype was compared between SLE and SLE_ONFH patients (Tables 4 and 5). Haplotype 3 (ht3: T-G-T-G-A) and haplotype 4 (ht4: C-C-T-A-A) were associated with an increased risk (OR; 3.73 ) of ONFH in SLE patients under recessive analysis model (Table 4). None of haplotypes located in block 2 showed evidence for association (data not shown). These results suggest that polymorphisms located in extracellular domain of CR2 gene may be functionally involved with increased susceptibility to ONFH in SLE patients.

\section{Discussion}

Although ONFH is a common complication deteriorating the treatment of SLE, details of the pathogenesis are not well 


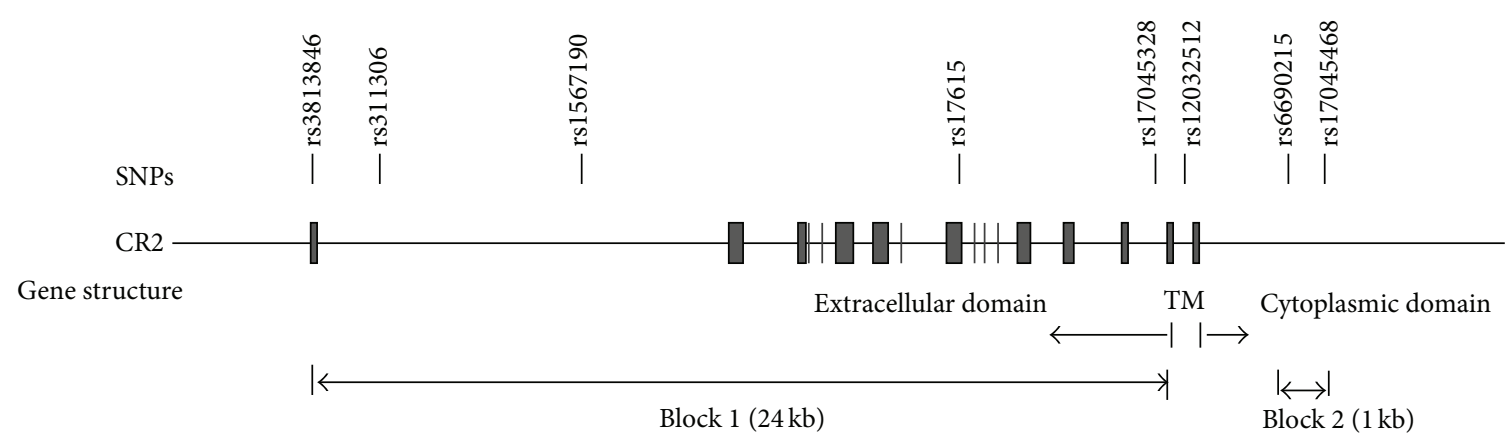

(a)

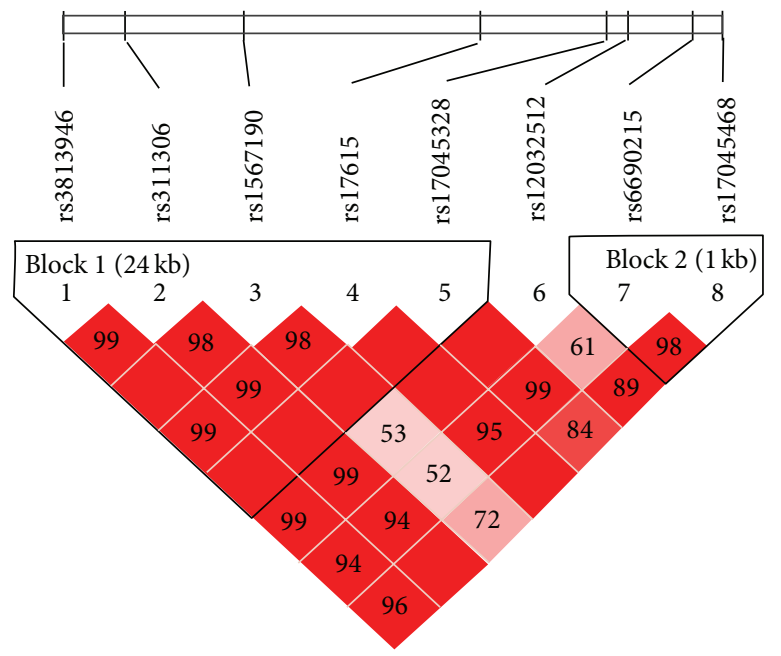

(b)

FIgURE 1: CR2 SNP locations and haplotype blocks. (a) The CR2 gene consists of 20 exons. Eight SNPs in the $5^{\prime}$ UTR, exon 10, and several intron regions were genotyped. (b) $D^{\prime}$ and $r^{2}$ of each SNP pair are shown. Two haplotype blocks were constructed based on the strength of LD among SNP pairs. The first 5 SNPs formed $24 \mathrm{~kb}$ block 1 and next SNPs formed block 2 (see Table 5).

TABLE 1: List of TaqMan probes for SNP genotyping of CR2 SNPs.

\begin{tabular}{|c|c|c|}
\hline $\begin{array}{l}\text { TaqMan genotyping } \\
\text { SNP ID }\end{array}$ & Probes (ABI) & Context sequence [VIC/FAM] \\
\hline rs3813946 & C_25599654_10 & CTCACAGCTGCTTGCTGCTCCAGCC[C/T]TGCCCTCCCAGAGCTGCCGGACGCT \\
\hline rs311306 & C_1009912_10 & ACCTATCACCATCTAAAACCTTCTT[ $\underline{\overline{\mathrm{C} / \mathrm{G}]}} \mathrm{CTTATTTATGTACCTGTTTGTTGTT}$ \\
\hline rs1567190 & C_8827000_20 & GGAGTATAGGCTACATAGTGAGGAA[C/T]GGTAGTTGATTAAATGGATTGGAGC \\
\hline rs17615 & C_12082973_1_ & TATAGTGGATTTACTTTGAAGGGCA $\underline{\overline{[\mathbf{A} / \mathbf{G}}]}$ TAGTCAGATTCGTTGCAAAGCTGAT \\
\hline rs17045328 & C_32843029_10 & ATTCTAACCTGAGAAATCTCTGATT[A/G]TAAAGTTGAGTATATTGTTTTTGTC \\
\hline rs12032512 & C_26228419_10 & AACCTTAAGCTCAACTATGATTATT[C/G]AGGAATTCAGCATTTATGTCCAAGA \\
\hline rs6690215 & C_30168452_10 & TTGGTGAGGATGCAAAGCAAATGGT[C/T]AATATTTGGGAGTTTTAATCAGGAA \\
\hline rs17045468 & C_32842997_10 & 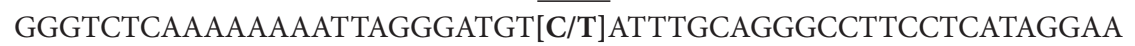 \\
\hline
\end{tabular}

VIC: $2^{\prime}$-chloro-7' -phenyl-1,4-dichloro-6-carboxyfluorescein. VIC is a fluorescent dye that was originally developed by Applied Biosystems. FAM: 6-carboxyfluorescein.

established. Because venous thrombosis and resultant blood flow obstruction mediated by thrombophilia or hypofibrinolysis are generally assumed to develop ONFH $[18,19]$, most of gene studies have focused on gene polymorphisms affecting the coagulation and fibrinolytic systems $[8,9]$. Recent studies, however, reported that immunologic factors might develop
ONFH $[6,7]$ and few genetic studies were performed to reveal their roles in the development of SLE_ONFH [10].

Complement receptor type 2 (CR2) is a membrane glycoprotein that binds $\mathrm{C} 3$ degradation products generated during complement activation, specifically iC3b, C3dg, and C3d. It has many important functions in normal immunity, such as 
TABLE 2: SNP markers in the CR2 gene genotyped in this case-control study.

\begin{tabular}{|c|c|c|c|c|c|c|c|c|}
\hline \multirow{2}{*}{$\begin{array}{l}\text { rs number } \\
\text { (alternative name) }\end{array}$} & \multirow{2}{*}{ Position } & \multirow{2}{*}{ Amino acid substitution } & \multicolumn{3}{|c|}{ Genotype } & \multicolumn{2}{|c|}{$\mathrm{MAF}^{(1)}$} & \multirow[t]{2}{*}{$\mathrm{HWE}^{(2)}$} \\
\hline & & & $\mathrm{C} / \mathrm{C}^{(3)}$ & $\mathrm{C} / \mathrm{R}$ & $\mathrm{R} / \mathrm{R}$ & Control & Case & \\
\hline $\begin{array}{l}\text { rs3813946 } \\
(\mathrm{T} / \mathrm{C})\end{array}$ & Exon $\left(5^{\prime}\right.$ UTR $)$ & No & $\mathrm{TT}$ & $\mathrm{CT}$ & $\mathrm{CC}$ & 0.144 & 0.184 & 0.309 \\
\hline $\begin{array}{l}\text { rs311306 } \\
\text { (G/C) }\end{array}$ & Intron 1 & No & GG & CG & CC & 0.139 & 0.20 & 0.299 \\
\hline $\begin{array}{l}\text { rs } 1567190 \\
(\mathrm{~T} / \mathrm{C})\end{array}$ & Intron 1 & No & $\mathrm{TT}$ & CT & $\mathrm{CC}$ & 0.419 & 0.34 & 0.864 \\
\hline $\begin{array}{l}\text { rs17615 } \\
\text { (G/A) }\end{array}$ & Coding exon 10 & Ser639Asn (AGT-AAT) & GG & AG & AA & 0.133 & 0.163 & 0.077 \\
\hline $\begin{array}{l}\text { rs17045328 } \\
\text { (A/G) }\end{array}$ & Intron 16 & No & $\mathrm{AA}$ & AG & GG & 0.262 & 0.208 & 0.517 \\
\hline $\begin{array}{l}\text { rs12032512 } \\
(\mathrm{C} / \mathrm{G})\end{array}$ & Intron 17 & No & $\mathrm{CC}$ & CG & GG & 0.419 & 0.45 & 0.396 \\
\hline $\begin{array}{l}\text { rs6690215 } \\
(\mathrm{C} / \mathrm{T})\end{array}$ & Intron 18 & No & $\mathrm{CC}$ & $\mathrm{CT}$ & $\mathrm{TT}$ & 0.463 & 0.43 & 0.618 \\
\hline $\begin{array}{l}\text { rs17045468 } \\
(\mathrm{C} / \mathrm{T})\end{array}$ & Intron 18 & No & $\mathrm{CC}$ & $\mathrm{CT}$ & $\mathrm{TT}$ & 0.255 & 0.23 & 0.379 \\
\hline
\end{tabular}

${ }^{(1)}$ MAF: minor allele frequency; ${ }^{(2)}$ HWE: $P$ values of deviation from Hardy-Weinberg equilibrium.

${ }^{(3)} \mathrm{C} / \mathrm{C}$ : major homozygote; C/R: heterozygote; R/R: minor homozygote.

TABLE 3: Analyses of association between CR2 gene polymorphisms and the risk of ONFH in SLE patients.

\begin{tabular}{|c|c|c|c|c|c|c|c|c|c|}
\hline \multirow{2}{*}{\multicolumn{2}{|c|}{ SNP rs number Genotype }} & \multicolumn{2}{|c|}{ Frequencies (\%) } & \multicolumn{2}{|c|}{ Allele } & \multicolumn{2}{|c|}{ Dominant } & \multicolumn{2}{|c|}{ Recessive } \\
\hline & & Controls (SLE) & Cases (SLE_ONFH) & OR (95\% CI) & $P$ & OR $(95 \% \mathrm{CI})$ & $P$ & OR (95\% CI) & $P$ \\
\hline \multirow{3}{*}{ rs3813946 } & TT & $102(71.83)$ & $35(71.43)$ & & & & & & \\
\hline & CT & $39(27.46)$ & $10(20.41)$ & $1.33(0.73-2.45)$ & 0.353 & $1.02(0.50-2.10)$ & 0.957 & $12.5(1.37-115)$ & $0.016^{*}$ \\
\hline & $\mathrm{CC}$ & $1(0.7)$ & $4(8.16)$ & & & & & & \\
\hline \multirow{3}{*}{ rs311306 } & GG & $108(72.97)$ & $35(70.0)$ & & & & & & \\
\hline & CG & $39(26.35)$ & $10(20.0)$ & $1.56(0.86-2.81)$ & 0.141 & $1.16(0.57-2.34)$ & 0.685 & $16.3(1.86-143)$ & $0.004^{*}$ \\
\hline & $\mathrm{CC}$ & $1(0.68)$ & $5(10.0)$ & & & & & & \\
\hline \multirow{3}{*}{ rs1567190 } & TT & 49 (33.11) & $22(44.0)$ & & & & & & \\
\hline & CT & $74(50.0)$ & $22(44.0)$ & $0.72(0.45-1.15)$ & 0.164 & $0.63(0.33-1.21)$ & 0.165 & $0.67(0.26-1.74)$ & 0.411 \\
\hline & $\mathrm{CC}$ & $25(16.89)$ & $6(12.0)$ & & & & & & \\
\hline \multirow{3}{*}{ rs17615 (S639N) } & GG & $108(73.47)$ & $36(73.47)$ & & & & & & \\
\hline & $\mathrm{AG}$ & $39(26.53)$ & $10(20.41)$ & $1.28(0.68-2.40)$ & 0.450 & $1.0(0.48-2.08)$ & 1 & & $0.015^{*}$ \\
\hline & $\mathrm{AA}$ & $0(0)$ & $3(6.12)$ & & & & & & \\
\hline \multirow{3}{*}{ rs17045328 } & $\mathrm{AA}$ & $78(53.06)$ & $31(64.58)$ & & & & & & \\
\hline & AG & $61(41.5)$ & $14(29.17)$ & $0.74(0.43-1.29)$ & 0.292 & $0.62(0.32-1.22)$ & 0.163 & $1.16(0.30-4.55)$ & $0.734^{*}$ \\
\hline & GG & $8(5.44)$ & $3(6.25)$ & & & & & & \\
\hline \multirow{3}{*}{ rs12032512 } & $\mathrm{CC}$ & $47(31.76)$ & $16(32.0)$ & & & & & & \\
\hline & CG & $78(52.7)$ & $23(46.0)$ & $1.14(0.72-1.79)$ & 0.587 & $0.99(0.50-1.97)$ & 0.975 & $1.53(0.69-3.42)$ & 0.295 \\
\hline & GG & $23(15.54)$ & $11(22.0)$ & & & & & & \\
\hline \multirow{3}{*}{ rs6690215 } & $\mathrm{CC}$ & $41(27.7)$ & $20(40.0)$ & & & & & & \\
\hline & $\mathrm{CT}$ & 77 (52.03) & $17(34.0)$ & $0.88(0.55-1.38)$ & 0.569 & $0.58(0.29-1.12)$ & 0.103 & $1.38(0.65-2.92)$ & 0.396 \\
\hline & TT & $30(20.27)$ & $13(26.0)$ & & & & & & \\
\hline \multirow{3}{*}{ rs17045468 } & $\mathrm{CC}$ & $79(53.74)$ & $30(60.0)$ & & & & & & \\
\hline & CT & $61(41.5)$ & $17(34.0)$ & $0.87(0.51-1.49)$ & 0.616 & $0.78(0.40-1.49)$ & 0.442 & $1.28(0.32-5.14)$ & $0.716^{*}$ \\
\hline & TT & $7(4.76)$ & $3(6.0)$ & & & & & & \\
\hline
\end{tabular}

Genotype distributions are shown as number (\%). Chi-square $P$ values and odds ratio (95\% CI) are shown. OR: odds ratio; CI: confidence interval.

*Fisher's exact test. 
TABLE 4: Analyses of association between CR2 gene haplotypes and the risk of ONFH in SLE patients.

\begin{tabular}{|c|c|c|c|c|c|c|c|c|c|}
\hline \multirow{2}{*}{ Haplotype } & \multirow{2}{*}{ Genotype } & \multicolumn{2}{|c|}{ Frequencies (\%) } & \multicolumn{2}{|c|}{ Allele } & \multicolumn{2}{|c|}{ Dominant } & \multicolumn{2}{|c|}{ Recessive } \\
\hline & & SLE & SLE_ONFH & OR $(95 \% \mathrm{CI})$ & $P$ & OR $(95 \% \mathrm{CI})$ & $P$ & OR (95\% CI) & $P$ \\
\hline \multirow{3}{*}{$\begin{array}{l}\text { Block1-ht1 } \\
\text { T-G-C-G-A }\end{array}$} & $-/-$ & $46(32.86)$ & $22(45.83)$ & & & & & & \\
\hline & $h t 1 /-$ & $71(50.71)$ & $21(43.75)$ & $0.66(0.41-1.08)$ & 0.10 & $0.58(0.30-1.13)$ & 0.106 & $0.59(0.21-1.65)$ & 0.313 \\
\hline & $h t 1 / h t 1$ & $23(16.43)$ & $5(10.42)$ & & & & & & \\
\hline \multirow{3}{*}{$\begin{array}{l}\text { Block1-ht2 } \\
\text { T-G-T-G-G }\end{array}$} & $-/-$ & $74(52.86)$ & $31(64.58)$ & & & & & & \\
\hline & $h+2 /-$ & $58(41.43)$ & $14(29.17)$ & $0.73(0.42-1.28)$ & 0.275 & $0.62(0.31-1.21)$ & 0.158 & $1.1(0.28-4.33)$ & 1.0 \\
\hline & $h t 2 / h t 2$ & $8(5.71)$ & $3(6.25)$ & & & & & & \\
\hline \multirow{3}{*}{$\begin{array}{l}\text { Block1-ht3 } \\
\text { T-G-T-G-A }\end{array}$} & $-/-$ & $96(68.57)$ & $28(58.33)$ & & & & & & \\
\hline & $h t 3 /-$ & $38(27.14)$ & $13(27.08)$ & $1.8(1.05-3.09)$ & 0.031 & $1.56(0.79-3.06)$ & 0.196 & $3.81(1.21-12.0)$ & $0.023^{*}$ \\
\hline & $h t 3 / h t 3$ & $6(4.29)$ & $7(14.58)$ & & & & & & \\
\hline \multirow{3}{*}{$\begin{array}{l}\text { Block1-ht4 } \\
\text { C-C-T-A-A }\end{array}$} & $-/-$ & $101(72.14)$ & $35(72.92)$ & & & & & & \\
\hline & $h t 4 /-$ & $39(27.86)$ & $10(20.83)$ & $1.24(0.66-2.33)$ & 0.512 & $0.96(0.46-2.01)$ & 0.918 & & $0.016^{*}$ \\
\hline & $h t 4 / h t 4$ & $0(0.0)$ & $3(6.25)$ & & & & & & \\
\hline
\end{tabular}

(i) SNP order of haplotypes: rs3813946 (T/C)-rs311306 (G/C)-rs1567190 (T/C)-rs17615 (G/A)-rs17045328 (A/G).

(ii) Haplotype distributions are shown as number (\%). Chi-square $P$ values and odds ratio (95\% CI) are shown. OR: odds ratio; CI: confidence interval.

(iii) * Fisher's exact test.

TABLE 5: Haplotypes of CR2 in blocks 1 and 2 shown in Figure 1.

\begin{tabular}{lccc}
\hline Haplotype ID & & Haplotypes & Frequencies \\
\hline \multirow{3}{*}{ Block 1 } & $h t 1$ & TGCGA & 0.407 \\
& $h t 2$ & TGTGG & 0.255 \\
& $h t 3$ & TGTGA & 0.196 \\
& $h t 4$ & CCTAA & 0.142 \\
\hline \multirow{3}{*}{ Block 2 } & $h t 1$ & TC & 0.449 \\
& $h t 2$ & CC & 0.287 \\
& $h t 3$ & CT & 0.262 \\
\hline
\end{tabular}

targeting antigen to follicular dendritic cells in secondary lymphoid organs and cooperating with the $\mathrm{B}$ cell receptor to activate $\mathrm{B}$ cells [13]. CR2 is also assumed to play a role in the development of autoimmune disease [16]. Therefore, given these pleiotropic effects of complement receptor, we investigated whether polymorphisms of the CR2 gene were associated with the development of SLE_ONFH.

In this study, the SNPs rs3813946 in $5^{\prime}$-UTR, rs311306 in intron 1, and rs17615 in exon 10 (nonsynonymous SNP; G/A, Ser639Asn) of the CR2 gene are associated with the susceptibility of SLE_ONFH under recessive model. Haplotype T-G-T-G-A (ht3) and haplotype C-C-T-A-A (ht4) (SNP order of haplotypes: rs3813946-rs311306-rs1567190-rs17615rs17045328) were also associated with an increased risk (OR; 3.73 ) of SLE_ONFH (Table 4). However, when Bonferroni correction for multiple testing was applied, there was no significance in all SNPs and haplotypes. Previously, it was reported that the minor C allele of rs3813946, located in $5^{\prime}$ UTR of CR2, reduced transcription of reporter genes in CR2-nonexpressing erythroleukemia cells [13] and CR2 expressing B cells [16]. Under basic conditions, primary $\mathrm{B}$ cells from individuals homozygous or heterozygous for the minor allele at rs3813946 demonstrated a trend toward reduced levels of CR2 RNA transcript [16]. Nonsynonymous SNP rs17615, which is located in exon 10 of the CR2 gene, might also have functional effects that could lead to disease. Exon 10 is located directly in $5^{\prime}$ of alternatively spliced exon $10 \mathrm{a}$, which is found in a long CR2 isoform [20]. SNPs in coding region can change pre-mRNA splicing and message stability [21], and rs17615 allelic variant may regulate the relative level of the long and short isoform of CR2 [16]. Alternative splicing can be involved in the process of regulating normal physiological functions as well as pathologies. Genomewide alternative splicing studies estimate that greater than $95 \%$ of human multiexon genes express multiple splice isoforms. Interindividual variation in isoforms resulting from SNPs located in splicing regulatory motifs can occur in up to $21 \%$ of alternatively spliced genes [22], and the effects of these on splicing efficiency are assumed to contribute significantly to disease severity as well as susceptibility [23]. Alterations of CR2 expression have variable different effects on manifestations of disease in animal models of autoimmunity [24]. In addition, the substitution of asparagine for serine (rs17615; Ser639Asn), which is conserved in mice, rats, and sheep, may be important in receptor function. Therefore, there is a possibility that the dysregulation expression of CR2 is associated with the occurrence of the SLE_ONFH. Although the current study showed positive relationship between CR2 polymorphisms and SLE_ONFH, it is also limited. First, we had limited basic and clinical data of study samples. Important information such as family history of ONFH, onset of diseases, and medication history was not available in this study. Second, our study sample size is not enough for analysis of the effect of CR2 gene in ONFH with SLE. Although ONFH is one of the most common diseases around the hip joint in Korea, the incidence is relatively low in most countries. According to medical claims data from Korean National Health Insurance Corporation, the estimated average number of annual prevalent cases was 28.9 per 100,000 . Moreover, 
the incidence of ONFH in SLE patients is very low. We did not get enough samples for obtaining high power in the association analysis. Nevertheless, we believe that our findings are valuable because this is first study to reveal the association between polymorphisms of the CR2 gene and susceptibility of SLE_ONFH. This study will promote the replication study of other researchers on the suggestive results and thus will improve our understanding of $\mathrm{ONFH}$ pathogenesis in SLE patients.

In conclusion, three CR2 polymorphisms are associated with ONFH susceptibility in SLE patients in our case-control study and these findings may provide evidence that CR2 contributes to human ONFH susceptibility in SLE patients. However, further well-designed studies with large sample size are mandatory for establishing our findings and reveal clinical importance of them.

\section{Competing Interests}

The authors do not have any competing interests.

\section{Acknowledgments}

This work was supported by Biomedical Research Institute grant, Kyungpook National University Hospital (2015).

\section{References}

[1] Y. Assouline-Dayan, C. Chang, A. Greenspan, Y. Shoenfeld, and M. E. Gershwin, "Pathogenesis and natural history of osteonecrosis," Seminars in Arthritis and Rheumatism, vol. 32, no. 2, pp. 94-124, 2002.

[2] M. Abeles, J. D. Urman, and N. F. Rothfield, "Aseptic necrosis of bone in systemic lupus erythematosus. Relationship to corticosteroid therapy," Archives of Internal Medicine, vol. 138, no. 5 , pp. $750-754,1978$.

[3] M. A. Mont, C. J. Glueck, I. H. Pacheco, P. Wang, D. S. Hungerford, and M. Petri, "Risk factors for osteonecrosis in systemic lupus erythematosus," Journal of Rheumatology, vol. 24, no. 4, pp. 654-662, 1997.

[4] E. E. Velayos, J. D. Leidholt, C. J. Smyth, and R. Priest, "Arthropathy associated with steroid therapy," Annals of Internal Medicine, vol. 64, no. 4, pp. 759-771, 1966.

[5] G. H. Leventhal and H. D. Dorfman, "Aseptic necrosis of bone in systemic lupus erythematosus," Seminars in Arthritis and Rheumatism, vol. 4, no. 1, pp. 73-93, 1974.

[6] M. G. Tektonidou and H. M. Moutsopoulos, "Immunologic factors in the pathogenesis of osteonecrosis," Orthopedic Clinics of North America, vol. 35, no. 3, pp. 259-263, 2004.

[7] M. N. Weitzmann and R. Pacifici, "The role of T lymphocytes in bone metabolism," Immunological Reviews, vol. 208, pp. 154$168,2005$.

[8] J.-D. Chang, M. Hur, S.-S. Lee, J.-H. Yoo, and K. M. Lee, "Genetic background of nontraumatic osteonecrosis of the femoral head in the Korean population," Clinical Orthopaedics and Related Research, vol. 466, no. 5, pp. 1041-1046, 2008.

[9] T.-H. Kim, S.-H. Baek, J. O. Lim, S.-H. Lee, and S.-Y. Kim, "Genetic variation in the coagulation factor $\mathrm{V}$ gene and risk of femoral head osteonecrosis," Molecular Medicine Reports, vol. 12, no. 3, pp. 4434-4440, 2015.
[10] H. S. Kim, S.-C. Bae, T.-H. Kim, and S.-Y. Kim, "Endothelial nitric oxide synthase gene polymorphisms and the risk of osteonecrosis of the femoral head in systemic lupus erythematosus," International Orthopaedics, vol. 37, no. 11, pp. 22892296, 2013.

[11] K. B. Douglas, D. C. Windels, J. Zhao et al., "Complement receptor 2 polymorphisms associated with systemic lupus erythematosus modulate alternative splicing," Genes and Immunity, vol. 10, no. 5, pp. 457-469, 2009.

[12] J. Zhao, B. M. Giles, R. L. Taylor et al., "Preferential association of a functional variant in complement receptor 2 with antibodies to double-stranded DNA," Annals of the Rheumatic Diseases, vol. 75, no. 1, pp. 242-252, 2016.

[13] M. N. Cruickshank, M. Karimi, R. L. Mason et al., "Transcriptional effects of a lupus-associated polymorphism in the 5' untranslated region (UTR) of human complement receptor 2 (CR2/CD21)," Molecular Immunology, vol. 52, no. 3-4, pp. 165173, 2012.

[14] V. M. Holers, "Complement receptors and the shaping of the natural antibody repertoire," Springer Seminars in Immunopathology, vol. 26, no. 4, pp. 405-423, 2005.

[15] M. C. Hochberg, "Updating the American College of Rheumatology revised criteria for the classification of systemic lupus erythematosus," Arthritis and Rheumatism, vol. 40, no. 9, p. $1725,1997$.

[16] H. Wu, S. A. Boackle, P. Hanvivadhanakul et al., "Association of a common complement receptor 2 haplotype with increased risk of systemic lupus erythematosus," Proceedings of the National Academy of Sciences of the United States of America, vol. 104, no. 10, pp. 3961-3966, 2007.

[17] H. Miyagawa, M. Yamai, D. Sakaguchi et al., "Association of polymorphisms in complement component $\mathrm{C} 3$ gene with susceptibility to systemic lupus erythematosus," Rheumatology, vol. 47, no. 2, pp. 158-164, 2008.

[18] L. C. Jones, M. A. Mont, T. B. Le et al., "Procoagulants and osteonecrosis," The Journal of Rheumatology, vol. 30, no. 4, pp. 783-791, 2003.

[19] M. A. Kerachian, E. J. Harvey, D. Cournoyer, T. Y. K. Chow, and C. Séguin, "Avascular necrosis of the femoral head: vascular hypotheses," Endothelium, vol. 13, no. 4, pp. 237-244, 2006.

[20] Y.-J. Liu, J. Xu, O. de Bouteiller et al., "Follicular dendritic cells specifically express the long CR2/CD21 isoform," Journal of Experimental Medicine, vol. 185, no. 1, pp. 165-170, 1997.

[21] L. E. Maquat, "Evidence that selenium deficiency results in the cytoplasmic decay of GPx1 mRNA dependent on pre-mRNA splicing proteins bound to the mRNA exon-exon junction," BioFactors, vol. 14, no. 1-4, pp. 37-42, 2001.

[22] T. Kwan, D. Benovoy, C. Dias et al., "Genome-wide analysis of transcript isoform variation in humans," Nature Genetics, vol. 40, no. 2, pp. 225-231, 2008.

[23] G.-S. Wang and T. A. Cooper, "Splicing in disease: disruption of the splicing code and the decoding machinery," Nature Reviews Genetics, vol. 8, no. 10, pp. 749-761, 2007.

[24] C. J. Del Nagro, R. V. Kolla, and R. C. Rickert, "A critical role for complement C3d and the B cell coreceptor (CD19/CD21) complex in the initiation of inflammatory arthritis," The Journal of Immunology, vol. 175, no. 8, pp. 5379-5389, 2005. 

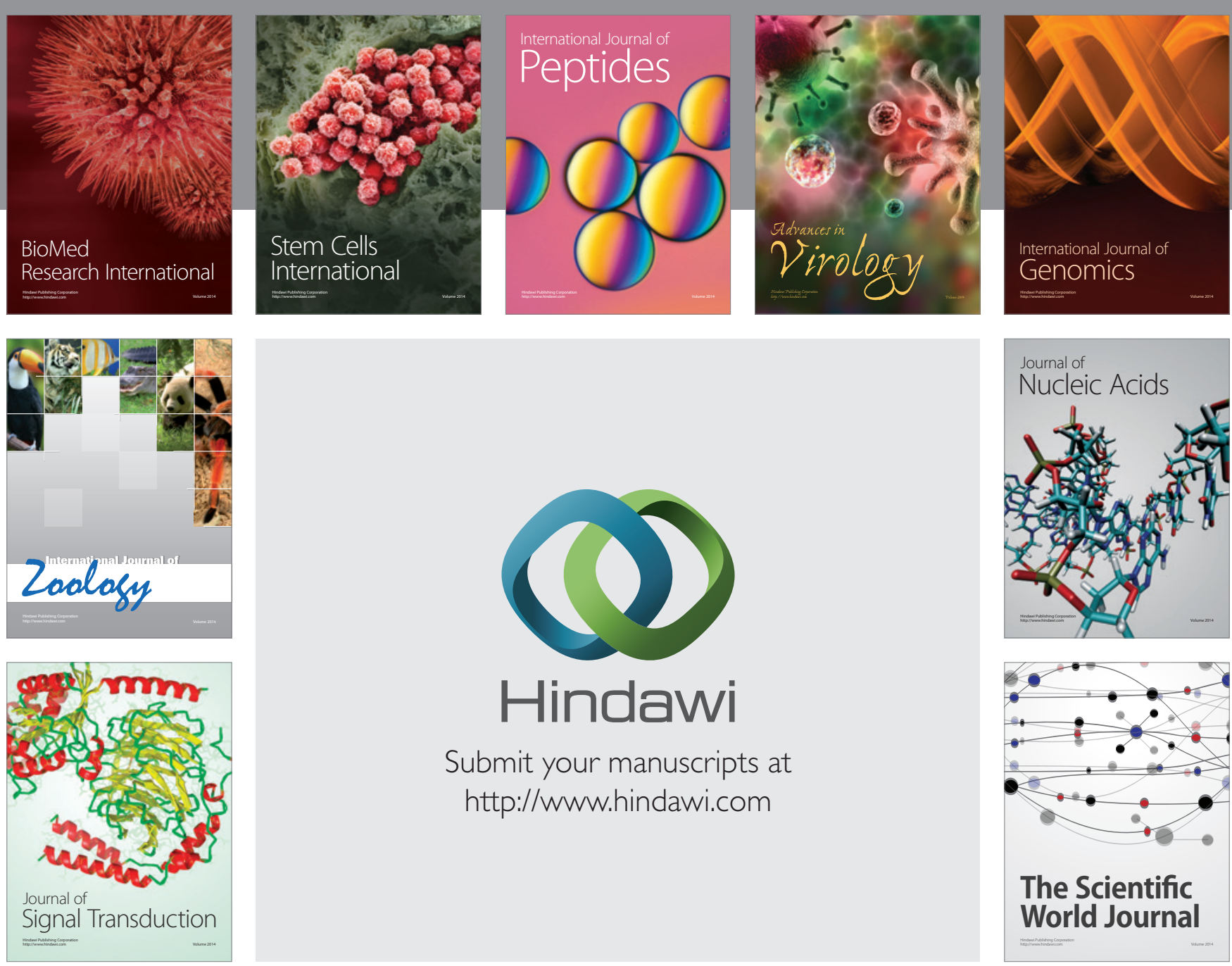

Submit your manuscripts at

http://www.hindawi.com
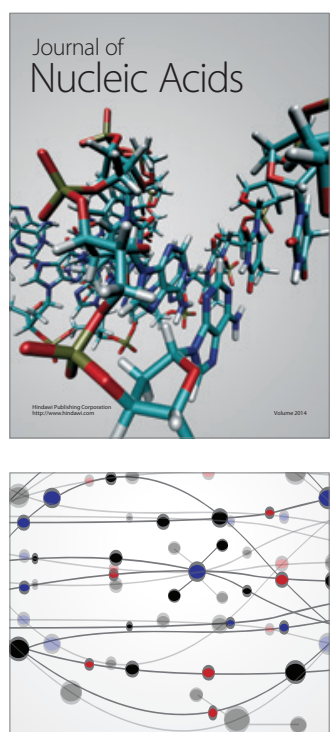

The Scientific World Journal
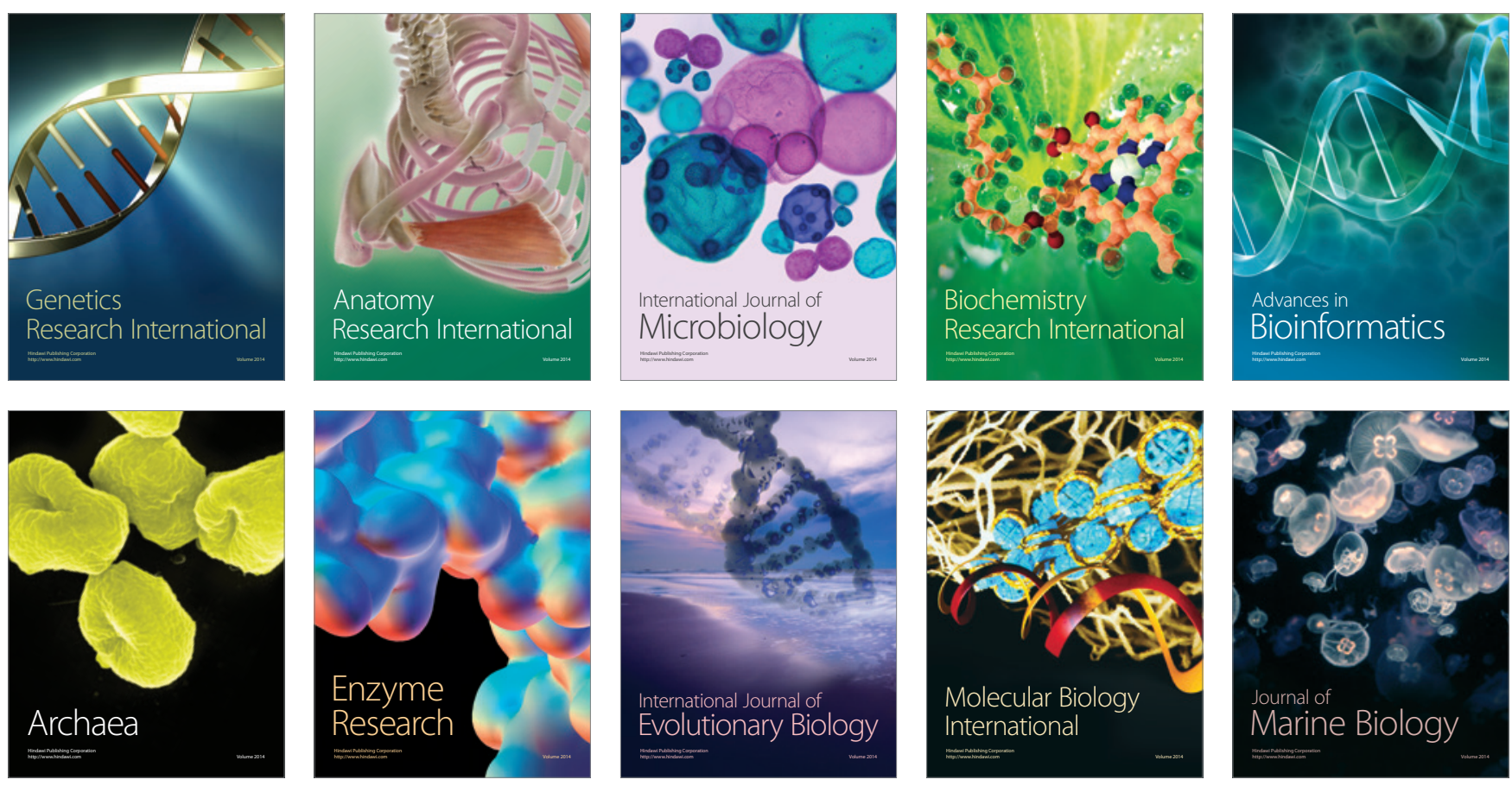